\title{
Prognostic significance of differentiating necrosis from fluid collection on endoscopic ultrasound in patients with presumed isolated extrapancreatic necrosis
}

\author{
Surinder S. Rana ${ }^{a}$, Puneet Chhabra ${ }^{a *}$, Ravi Sharma ${ }^{a}$, Vishal Sharma ${ }^{a}$, Rajesh Guptab, Deepak K. Bhasin ${ }^{a *}$ \\ Post Graduate Institute of Medical Education and Research (PGIMER), Chandigarh, India
}

Abstract

Departments of ${ }^{a}$ Gastroenterology (Surinder S. Rana, Puneet Chhabra, Ravi Sharma, Vishal Sharma, Deepak K. Bhasin); 'burgery (Rajesh Gupta), Post Graduate Institute of Medical Education and Research (PGIMER), Chandigarh, India

${ }^{\star}$ Current affiliation: Fortis Hospital, Mohali, India

Conflict of Interest: None

Correspondence to: Dr. Surinder S. Rana, MD, DM, Department of Gastroenterology, Post Graduate Institute of Medical Education and Research (PGIMER), Sector 12, Chandigarh, 160012 India, Tel.: +91 172 2756555, Fax: +91 172 2744401,

e-mail: drsurinderrana@gmail.com

Presented at DDW 2015, Washington DC, USA

Received 7 August 2016; accepted 11 October 2016; published online

DOI: https://doi.org/10.20524/aog.2016.0106

\section{Introduction}

Acute pancreatitis (AP) is a complex acute inflammatory disorder of the pancreas with potentially catastrophic consequences. Its clinical course is usually mild in the majority of patients, with uneventful recovery [1]. However, in up to $20 \%$ of patients the attack may be severe and may lead to a number of local and systemic complications, including organ failure, prolonged hospital course, and significant mortality [1-4]. The factors that determine the severity of AP have not been completely understood, and it is therefore often difficult to predict its clinical course [1-4]. A better understanding of the disease over the last few decades has led to the identification of clinical, investigational and radiological factors that can help in predicting the clinical course of AP [1,4].

Of these prognostic variable, pancreatic necrosis has been shown to be one of the significant determinants of poor 
prognosis [5-7]. Studies have shown that the majority of patients who develop organ failure, or other complications of AP, have necrotizing pancreatitis, while patients with non-necrotizing interstitial AP usually have an uneventful recovery [5-7]. Recent studies have also identified a new entity of extrapancreatic necrosis alone, which is defined as necrosis of the peripancreatic tissue only on contrast-enhanced imaging studies, with a normally enhanced pancreatic gland [8-10]. The prognosis of patients with isolated extrapancreatic necrosis is better than that of patients with pancreatic necrosis, but worse than that of patients with acute interstitial pancreatitis [8-10].

Extrapancreatic necrosis is usually diagnosed on contrastenhanced computed tomography (CECT) as extrapancreatic changes that are more than simple fat stranding; both peripancreatic fluid collections and peripancreatic necrosis have similar appearances on CECT $[8,9]$. Endoscopic ultrasound (EUS) has been shown to be a better modality than CECT for differentiating fluid collections from solid-containing necrotic collections [11-13]. We have also demonstrated that EUS can detect pancreatic necrosis reliably [14]. As EUS provides highresolution images of the pancreas and surrounding areas, we hypothesized that EUS may be able to differentiate between peripancreatic fluid collections and peripancreatic necrosis in patients with presumed isolated extrapancreatic necrosis and that these two subtypes of isolated extrapancreatic necrosis may have different clinical courses and outcomes.

\section{Patients and methods}

This study is a retrospective analysis of a prospectively collected database that includes all the patients with AP from a large tertiary care hospital. The data of all the patients with AP seen in our unit from December 2012 to November 2014 were retrieved, and patients with isolated extrapancreatic necrosis who had undergone both EUS and CECT were identified. Patients who had no contraindication for EUS and presented to us within 5 days of the onset of symptoms were enrolled. Those with coronary artery disease, pre-existing cardiac disorder or respiratory disorder, malignancy, pregnancy, underlying chronic pancreatitis, pancreatic malignancy, and patients who refused consent for EUS were excluded. Informed consent was obtained from all patients prior to the EUS procedure and the study protocol was approved by the institute's ethics committee.

All the participants of this study were thoroughly interviewed and underwent clinical and laboratory examinations. The diagnosis of acute pancreatitis was based on suggestive clinical history (acute onset upper abdominal pain with or without radiation to the back, vomiting, constipation and obstipation), physical examination, and serum amylase elevated to greater than three times the upper limit of normal and/or positive abdominal imaging [1]. Hematological investigations, blood sugar, serum amylase, blood urea, serum creatinine, albumin, globulin, lipid profile, calcium, phosphate, arterial blood gas analysis and liver function tests were performed on the day of admission. The etiology of acute pancreatitis was based on the patient's history of alcohol and drug use, biochemical investigations, transabdominal ultrasonography, and radiological findings. Organ failure was described as the presence of respiratory failure $\left(\mathrm{PaO}_{2} / \mathrm{FiO}_{2}<300\right)$, renal failure (serum creatinine $>1.8 \mathrm{mg} / \mathrm{dL}$ ), or cardiovascular failure (blood pressure $<90 \mathrm{mmHg}$ and non-responsive to fluids) [1]. Involvement of more than one organ system was classed as multiorgan failure, while if the organ failure persisted for $>48 \mathrm{~h}$ it was labeled as persistent organ failure.

All patients diagnosed with acute pancreatitis underwent EUS on the day of admission, performed by either of two experienced endosonologists (SSR, DKB) using a radial echoendoscope (Pentax EG-3670 URK radial echoendoscope, Pentax Corp., Japan or GF-UE 160 radial echoendoscope, Olympus Corp., Japan) at a frequency of 7.5 MHz. EUS was performed with the patient in the left lateral decubitus position under conscious sedation with intravenous midazolam (2.5-5 mg). The uncinate process and head of the pancreas were evaluated from the duodenum, and the body and tail of the pancreas were evaluated from the stomach.

An abdominal CECT scan was performed in all patients 3 days after the onset of symptoms and the presence of pancreatic/extrapancreatic necrosis was recorded. Pancreatic necrosis was defined as focal or diffuse areas of non-enhanced pancreatic parenchyma on CECT, whereas extrapancreatic necrosis was defined as extrapancreatic morphological changes that were more than simple fat stranding $[1,9]$.

The EUS images were retrieved for patients with isolated extrapancreatic necrosis. Isolated extrapancreatic necrosis was diagnosed on CT when there were extrapancreatic changes as defined above, with complete enhancement of the pancreatic parenchyma. On EUS, isolated extrapancreatic necrosis was characterized into two subtypes:

1. Peripancreatic necrosis: peripancreatic heterogeneously echotextured areas (Fig. 1).

2. Peripancreatic fluid collection: peripancreatic predominantly anechoic areas with no significant echogenic areas (Fig. 2).

To differentiate between peripancreatic fluid collections and peripancreatic necrosis, the EUS images were read separately by two experienced endosonographers (SSR and DKB) who were blinded to the clinical data. In the event of disagreement between the two endosonographers, the images were reviewed jointly by both and their consensus opinion was recorded.

These two groups were compared in relation to various outcome measures: organ failure (including multiorgan failure and persistent organ failure), need for percutaneous or endoscopic or surgical interventions for local complications, and mortality.

\section{Statistical analysis}

The descriptive data were presented as percentages for categorical variables. Categorical variables were compared using the chi-square test. A P-value of $<0.05$ was considered as significant. As this study was restricted to a small number of planned comparisons of outcomes, the Bonferroni correction was not applied. 

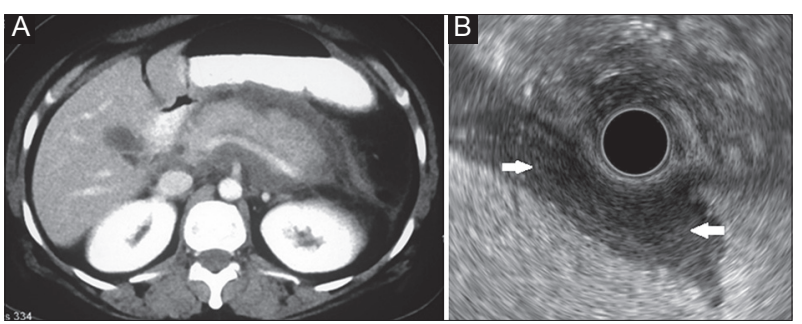

Figure 1 (A) Contrast-enhanced computed tomography showing isolated extrapancreatic necrosis with normally enhancing parenchyma. (B) Endoscopic ultrasound showing heterogeneous echotextured peripancreatic area suggestive of peripancreatic necrosis (arrows)
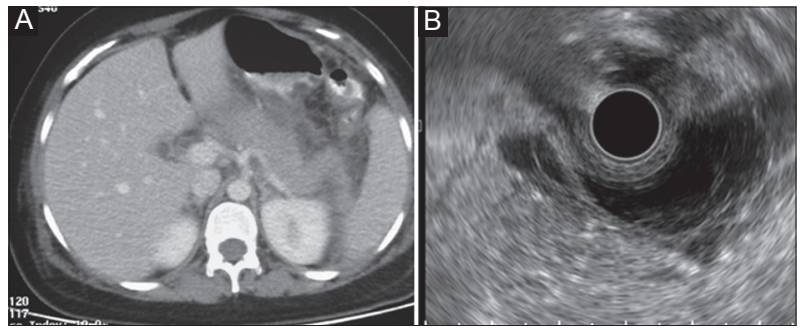

Figure 2 (A) Contrast-enhanced computed tomography showing Isolated extrapancreatic necrosis with normally enhancing parenchyma. (B) Endoscopic ultrasound showing anechoic areas around the pancreas suggestive of peripancreatic fluid collection (arrows)

\section{Results}

Thirty-six patients (25 male; age range 19-65 years) with isolated extrapancreatic necrosis (normally enhancing pancreatic parenchyma on CECT) were studied. The etiology of AP was alcohol in $16(44.4 \%)$ patients, gallstone disease in $13(36.1 \%)$, and other in $7(19.4 \%)$ patients. Twenty-eight (77.7\%) patients developed pleural effusion, 6 (16.6\%) developed ascites, 20 (55.5\%) developed organ failure, 13 (36.1\%) developed persistent organ failure, 3 (8.3\%) developed multiorgan failure, 5 (14\%) needed endoscopic/ percutaneous radiological intervention, 2 (5.5\%) needed surgery and $2(5.5 \%)$ patients succumbed to the illness.

On EUS, $25(69.4 \%)$ patients had peripancreatic heterogeneously echotextured areas suggestive of peripancreatic necrosis and $11(30.6 \%)$ patients had peripancreatic anechoic areas suggestive of peripancreatic fluid collections. None of the patients had EUS features of pancreatic necrosis, as described in our previous paper [14]. There was no significant difference between the demographic profiles of patients in these two groups (Table 1).

\section{Clinical outcomes (Table 1)}

Of the 25 patients with peripancreatic necrosis, 22 (88\%) developed pleural effusion, 5 (20\%) developed ascites, 17 (68\%) had organ failure, $12(48 \%)$ had persistent organ failure, and $3(12 \%)$ had multiorgan failure. On follow
Table 1 Comparison of outcomes in patients with peripancreatic necrosis and peripancreatic fluid collections

\begin{tabular}{lccc}
\hline Variable & $\begin{array}{c}\text { Peripancreatic } \\
\text { necrosis } \\
(\mathrm{N}=25) \\
\mathrm{N}(\%)\end{array}$ & $\begin{array}{c}\text { Peripancreatic } \\
\text { fluid } \\
\text { collection (N=11) }\end{array}$ & P-value \\
\hline N (\%) & \\
\hline Male:female & $18: 7$ & $7: 4$ & 0.70 \\
\hline Alcohol (etiology) & 10 & 6 & 0.48 \\
Gallstones (etiology) & 9 & 4 & 0.46 \\
\hline Pleural effusion & $22(88 \%)$ & $6(55 \%)$ & $\mathbf{0 . 0 4}$ \\
\hline Ascites & $5(20 \%)$ & $1(9 \%)$ & 0.64 \\
Organ failure & $17(68 \%)$ & $3(27 \%)$ & $\mathbf{0 . 0 3}$ \\
\hline MOF & $3(12 \%)$ & $0(0 \%)$ & 0.53 \\
POF & $12(48 \%)$ & $1(9 \%)$ & $\mathbf{0 . 0 3}$ \\
Intervention & $5(20 \%)$ & $0(0 \%)$ & 0.29 \\
\hline Surgery & $2(8 \%)$ & $0(0 \%)$ & 0.99 \\
\hline Mortality & $2(8 \%)$ & $0(0 \%)$ & 0.99 \\
\hline
\end{tabular}

MOF, multiple organ failure; $P O F$, persistent organ failure

up, $5(20 \%)$ patients needed endoscopic/radiological intervention, $2(8 \%)$ needed surgery and $2(8 \%)$ patients died. On the other hand, of 11 patients with peripancreatic fluid collection, 6 (55\%) developed pleural effusion, 1 (9\%) developed ascites, 3 (27\%) had organ failure, 1 (9\%) had persistent organ failure, and none had multiorgan failure. On follow up, none of the patients needed endoscopic/ radiological intervention or surgery and all patients recovered with no mortality.

Compared with patients who had peripancreatic fluid collection, the patients with peripancreatic necrosis had a significantly higher frequency of pleural effusion ( $88 \%$ vs. $55 \%$; $\mathrm{P}=0.04)$, organ failure (68\% vs. $27 \% ; \mathrm{P}=0.03)$, and persistent organ failure $(48 \%$ vs. $9 \% ; \mathrm{P}=0.03)$. The patients with peripancreatic necrosis also had a higher frequency of ascites ( $20 \%$ vs. 9\%), need for intervention ( $20 \%$ vs. nil), surgery ( $8 \%$ vs. nil) and mortality ( $8 \%$ vs. nil), but these differences were not statistically significant.

\section{Discussion}

In patients with acute necrotizing pancreatitis there is usually necrosis of the pancreatic parenchyma as well as the peripancreatic fat and tissues [1]. However, some patients have necrosis of the peripancreatic tissue/fat only, with a normally enhanced pancreatic gland on contrast-enhanced imaging studies; this entity has been labeled as isolated extrapancreatic necrosis. Previous studies have demonstrated that isolated extrapancreatic necrosis is an entity that is distinct from mild interstitial non-necrotizing pancreatitis and from pancreatic necrosis, alone or combined with extrapancreatic necrosis [8-10,15]. Patients with isolated extrapancreatic 
necrosis have been shown to have lower rates of persistent organ failure, local complications, need for intervention and mortality, compared to patients with combined pancreatic and extrapancreatic necrosis.

We have previously shown that EUS performed on the day of admission can accurately diagnose patients with acute necrotizing pancreatitis. On EUS, patients with acute necrotizing pancreatitis had novel findings of either multiple hypoechoic or hyperechoic areas measuring more than $5 \mathrm{~mm}$ in diameter, whereas these findings were not present in any of the patients with acute interstitial pancreatitis [14]. We also found that the presence of peripancreatic hypoechoic areas correlated with the presence of extrapancreatic necrosis on CT; these areas were found to be soft on EUS elastography, possibly suggesting fat necrosis [14]. However, not all patients who showed extrapancreatic necrosis on CT had similar findings on EUS, with some patients having anechoic peripancreatic areas suggestive of fluid collection, rather than hypoechoic areas that suggested peripancreatic necrosis. Therefore, in the current study we compared the clinical course of these two types of extrapancreatic necrosis.

In this EUS study, we have shown that presumed isolated extrapancreatic necrosis on CT can be characterized into two subtypes: peripancreatic necrosis, which is characterized by the presence of peripancreatic heterogeneously echotextured areas, and peripancreatic fluid collection, which is characterized by the presence of peripancreatic anechoic areas. Peripancreatic necrosis and peripancreatic fluid collections are associated with different clinical courses, with patients who have peripancreatic necrosis having higher rates of organ failure (including multiorgan failure and persistent organ failure), ascites, pleural effusion, need for percutaneous, endoscopic or surgical intervention for local complications, and mortality, when compared to patients with peripancreatic fluid collection. The higher frequency of complications in the peripancreatic necrosis group is not surprising, as the presence of tissue necrosis has been shown to be associated with a higher frequency of complications and organ failure $[5,16]$. The causes of organ failure and complications in AP are multifactorial and may possibly be due to the release of various cytokines and vasoactive substances, leading to the activation of proinflammatory pathways, as a consequence of pancreatic and peripancreatic tissue necrosis $[17,18]$.

Our study has a few limitations, including the small sample size and the exclusion of sick patients who had contraindications for EUS, such as those with hemodynamic or severe respiratory compromise. In addition, the interobserver variability was not assessed for either the CT or the EUS findings. Moreover, the EUS findings were not compared with the gold standard, namely surgical findings.

In conclusion, isolated extrapancreatic necrosis on CECT represents a heterogeneous group comprised of patients with peripancreatic fluid collections and/or peripancreatic necrosis. Patients who exhibit peripancreatic fluid collections on EUS have a less severe disease course than do those with peripancreatic necrosis.

\section{Summary Box}

\section{What is already known:}

- Pancreatic necrosis is one of the important determinants of poor prognosis in acute pancreatitis

- A new entity of extrapancreatic necrosis alone has been recognized, defined as necrosis of only the peripancreatic tissue on contrast-enhanced imaging studies, with a normally enhanced pancreatic gland

- The prognosis of patients with isolated extrapancreatic necrosis is better than that of those with pancreatic necrosis, but worse than that of those with acute interstitial pancreatitis

- Endoscopic ultrasound has been shown to be a better modality than contrast-enhanced computed tomography for differentiating fluid collections from solid-containing necrotic lesions

\section{What the new findings are:}

- Presumed isolated extrapancreatic necrosis on computed tomography can be characterized into two subtypes: peripancreatic necrosis, characterized by peripancreatic heterogeneously echotextured areas, and peripancreatic fluid collection, characterized by peripancreatic anechoic areas

- These subtypes have different clinical courses: patients who have peripancreatic necrosis have higher rates of organ failure, ascites, pleural effusion, need for percutaneous, endoscopic or surgical interventions for local complications, and mortality, compared to patients with peripancreatic fluid collection

\section{References}

1. Banks PA, Bollen TL, Dervenis C, et al; Acute Pancreatitis Classification Working Group. Classification of acute pancreatitis-2012: revision of the Atlanta classification and definitions by international consensus. Gut 2013;62:102-111.

2. Cappell MS. Acute pancreatitis: etiology, clinical presentation, diagnosis and therapy. Med Clin N Am 2008;92:889-923.

3. Nadkarni N, Bhasin DK, Rana SS, et al. Diastolic dysfunction, prolonged QTc interval and pericardial effusion as predictors of mortality in acute pancreatitis. J Gastroenterol Hepatol 2012;27:1576-1580.

4. Sharma V, Shanti Devi T, Sharma R, et al. Arterial pH, bicarbonate levels and base deficit at presentation as markers of predicting mortality in acute pancreatitis: a single-centre prospective study. Gastroenterol Rep (Oxf) 2014;2:226-231.

5. Garg PK, Madan K, Pande GK, et al. Association of extent and infection of pancreatic necrosis with organ failure and death 
in acute necrotizing pancreatitis. Clin Gastroenterol Hepatol 2005;3:159-166.

6. Lankisch PG, Struckmann K, Lehnick D. Presence and extent of extrapancreatic fluid collections are indicators of severe acute pancreatitis. Int J Pancreatol 1999;26:131-136.

7. Babu RY, Gupta R, Kang M, Bhasin DK, Rana SS, Singh R. Predictors of surgery in patients with severe acute pancreatitis managed by the step-up approach. Ann Surg 2013;257:737-750.

8. Sakorafas GH, Tsiotos GG, Sarr MG. Extrapancreatic necrotizing pancreatitis with viable pancreas: a previously under-appreciated entity. J Am Coll Surg 1999;188:643-648.

9. Bakker OJ, van Santvoort H, Besselink MG, et al. Extrapancreatic necrosis without pancreatic parenchymal necrosis: a separate entity in necrotising pancreatitis? Gut 2013;62:1475-1480.

10. Rana SS, Sharma V, Sharma RK, Chhabra P, Gupta R, Bhasin DK. Clinical significance of presence and extent of extrapancreatic necrosis in acute pancreatitis. $J$ Gastroenterol Hepatol 2015;30:794-798.

11. Rana SS, Bhasin DK. Should all fluid collections in delayed phase of acute necrotizing pancreatitis labeled as walled off pancreatic necrosis? Dig Dis Sci 2014;59:1338-1339.

12. Rana SS, Bhasin DK, Reddy YR, et al. Morphological features of fluid collections on endoscopic ultrasound in acute necrotizing pancreatitis: do they change over time? Ann Gastroenterol 2014;27:258-261.

13. Rana SS, Chaudhary V, Sharma R, Sharma V, Chhabra P, Bhasin DK. Comparison of abdominal ultrasound, endoscopic ultrasound and magnetic resonance imaging in detection of necrotic debris in walled-off pancreatic necrosis. Gastroenterol Rep (Oxf) 2016;4:50-53.

14. Rana SS, Bhasin DK, Sharma V, Sharma R, Chaudhary V, Chhabra P. Can early endoscopic ultrasound predict pancreatic necrosis in acute pancreatitis? Ann Gastroenterol 2014;27:404-408.

15. Wang M, Wei A, Guo Q, et al. Clinical outcomes of combined necrotizing pancreatitis versus extrapancreatic necrosis alone. Pancreatology 2016;16:57-65.

16. Larvin M, Chalmers AG, McMahon MJ. Dynamic contrast enhanced computed tomography: a precise technique for identifying and localising pancreatic necrosis. BMJ 1990;300:1425-1428.

17. Weber CK, Adler G. From acinar cell damage to systemic inflammatory response: current concepts in pancreatitis. Pancreatology 2001;1:356-362.

18. Makhija R, Kingsnorth AN. Cytokine storm in acute pancreatitis. $J$ Hepatobiliary Pancreat Surg 2002;9:401-410. 